\title{
A Smartwatch Centric Social Networking Application for Alzheimer People
}

\author{
Henda Chorfi Ouertani ${ }^{* 1}$, Ahlam Al-Mutairi ${ }^{1}$, Fatimah Al-Shehri ${ }^{1}$, GhadahAl-Hammad ${ }^{1}$, Maram Al-Suhaibani ${ }^{1}$, \\ Munira Al-Holaibah ${ }^{1}$, Nouf Al-Saleh ${ }^{1}$ \\ ${ }^{1}$ Information Technology Department, \\ College of Computer and Information Sciences, King Saud University \\ Riyadh, Saudi Arabia
}

\begin{abstract}
In recent years, people are increasingly interacting with an overwhelming of devices especially wearable devices. These later have initiated new ways in human connections with the world. The basic concept of these devices is that they can be worn instead of being carried. They have pervasive and unobtrusive presence. Furthermore, they can be used for helping people with serious medical conditions, like the Alzheimer people. In this context, an application that allows Alzheimer relatives to locate and track the patient by connecting the application with GPS smartwatch that is worn by the patient is proposed. A safety area is predefined by the relative in order to be notified once the patient gets beyond this area. When there is no connection with the GPS watch for any reason and the patient cannot be tracked, the relatives can send a broadcast message to all application users who wish to help and participate in such social and humanitarian work.
\end{abstract}

Keywords-Smartwatch; Alzheimer; broadcast; tracking; social application

\section{INTRODUCTION}

Alzheimer's disease is the most common cause of dementia. The word dementia describes a set of symptoms that can include memory loss and difficulties with thinking, problem-solving or language [1]. Nowadays Alzheimer has been growing frighteningly. In a matter of times, patients may forget where they have been, what they were doing, forgetting their loved one, or even worse they might forget who they are. Alzheimer's gets worse over time. The disease may cause a person to become confused, lost in familiar places, misplace things or have trouble with language [2]. Therefore, most of them depend on their family in all daily activities even though to help them getting back to home.

In another side, People are increasingly interacting with an overwhelming of devices including smartwatches, smartphones, and tablets. Wearable devices have initiated new capabilities in human interactions with the world. They are providing among other functionalities, tracking capabilities. The new technologies are also changing the social values. The advent of social networking increases the ability to stay in contact with friends and family. In an interconnected information society, people are willing to help.

In this paper, a social networking application, Locate-me, that combines the potential of wearable devices and social networking applications is proposed. It permits to locate and track an Alzheimer patient wearing a GPS watch. Locate-me enables relatives to make use of advanced location tools in a matter of moments and be notified for any changes that can happen. Furthermore, when there is no connection with the GPS watch for any reason and the patient cannot be tracked, the relatives can benefit of the social networking capabilities of the application and send a broadcast message to all users who wish to help and participate in such social and humanitarian work.

The remaining part of this paper is organized as follows. In Section 2, the literature is investigated and a study of the related applications to Locate $\mathrm{Me}$ is proposed. Section 3 is dedicated to the wearable devices with a main focus on smartwatches. Section 4 presents a detailed description of the application. Sections 5 and 6, respectively deals with the system implementation and its functioning. Section 7 concludes the work and presents the future directions.

\section{LITERATURE REVIEW}

The literature is investigated in order to study the particularities of the similar applications related to helping and tracking Alzheimer people. The most similar applications to the specificities of Locate-me are selected. Below, a brief description of each application is given. Afterward, a comparison based on the main features that needed to be present in such applications is provided. Those features were determined based on questionnaire conducted among people concerned by the Alzheimer disease.

- PocketFinder is a GPS tracking application which is available for free downloading for IOS and android platforms. It is designed to stay connected with kids, senior parents especially Alzheimer's and special needs loved ones [3].

- Tweri is a mobility solution for the families and the caregivers of the Alzheimer's patients that provide them a way to keep track and know where the patient is, in case they go beyond the safety limits or don't come back home at the scheduled time. It's help to improve the autonomy of Alzheimer's patients [4].

- Through Alzheimer's Association Comfort Zone Check-In, powered by Omnilink, caregivers and family members can provide individual's with Alzheimer's a greater level of independence, while still maintaining their safety and security. Comfort Zone Check-In is 
extremely easy to use enabling almost any individual or family to begin making use of advanced location tools in a matter of moments [5].

- SafetySnapp is a comprehensive tool to make sure your loved ones reach their destination safely. Be notified each day to take a picture that could make all the difference in an emergency situation. Keep tabs on those you care about most through GPS tracking on their device. Important information and an updated photo can be sent to emergency contacts instantly when needed [6].

TABLE I. APPLICATIONS COMPARISON

\begin{tabular}{|c|c|c|c|c|c|c|c|c|}
\hline Features & $\underset{t}{\mathscr{L}}$ & 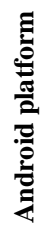 & 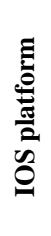 & ڤ్ర & 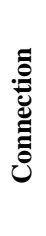 & 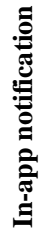 & 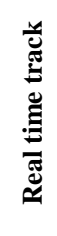 & 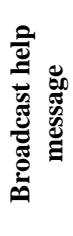 \\
\hline PocketFinder & $\checkmark$ & $\checkmark$ & $\checkmark$ & $\checkmark$ & $\checkmark$ & $\checkmark$ & $\checkmark$ & $\mathbf{X}$ \\
\hline Tweri & $\checkmark$ & $\checkmark$ & $\checkmark$ & $\checkmark$ & $\checkmark$ & $\checkmark$ & $\checkmark$ & $\mathbf{X}$ \\
\hline Comfort Zone & $\mathbf{X}$ & $\mathbf{X}$ & $\mathbf{X}$ & $\checkmark$ & $\checkmark$ & $\checkmark$ & $\checkmark$ & $\mathbf{X}$ \\
\hline SafetySnapp & $\checkmark$ & $\mathbf{X}$ & $\checkmark$ & $\checkmark$ & $\checkmark$ & $\checkmark$ & $\checkmark$ & $\checkmark$ \\
\hline Locate Me & $\checkmark$ & $\checkmark$ & $\mathbf{X}$ & $\checkmark$ & $\checkmark$ & $\checkmark$ & $\checkmark$ & $\checkmark$ \\
\hline
\end{tabular}

Based on the Table I above, it is clearly shown that all the applications support GPS, need internet connection to work, has in-app notification, do real time tracking, provide help for users and most of them are free. From the Table I, it is also noticed that only one of the four applications provides the feature of sending a help broadcast message which will be as a main feature in Locate-me application.

\section{WHY WATCH ?}

Nowadays, there is a wide range of different technologies that can be adapted and used for Alzheimer people, to help them maintain their independence and improve their quality of life.

People react differently to different assistive technologies. The nature of dementia may make people thoughtful and apprehensive of trying new devices. Therefore, certain user requirements should be taken into consideration, according to Cahill et al. [7]:

- The new device/product should fulfill the individual and formal caregiver needs,

- The design of the product is important, focusing on its familiarity and the fact that no new learning should be required on the part of the person with a dementia,

- A comprehensive assessment of needs should take place ideally at home with a health service professional fully trained in dementia care,
- Pre-testing is critical to ensure that the chosen device/product is reliable and effective, and it is important to find a product that suits the individual and is not complex or stigmatizing.

A smartwatch has the advantage of always being with the user, having ubiquitous and non-intrusive presence. Its design - being a wristwatch - is quite familiar, and its use is not complex or stigmatizing [8]. A smartwatch has many attractive features as any wearable computer. When implementing the application A Sony smart watch3 is used. It has the following features among others:

- Enjoy stylish design and monitor your phone at a glance.

- Control your device with voice and make calls handsfree.

- See messages on your phone automatically as they arrive.

- Capture photos, video and audio quickly on the go.

- Built-in GPS and a range of Health features to help you stay motivated while on the move. [9]

\section{APPLICATION DESCRIPTION}

Locate-me is a mobile application that enable Alzheimer' relatives to make use of advanced assistive technologies in order to track an Alzheimer person. The tracking is nonintrusive. Locate-Me permits relatives to give more autonomy to the Alzheimer person in a safe way.

Locate-me is targeting two types of users:

- The first target users are the patients' relatives. The application will allow them to locate and track the patient by connecting the application with GPS watch that is worn by the patient. A safety area is predefined by the relative in order to be informed once the patient gets out this area. When there is no connection with the GPS watch for any reason and the patient cannot be tracked, the patients' relatives can send a broadcast message to all application users in a specific city. This message will be a request for help to find the patient. The relative can include in his message: patient photo, description. Furthermore, the relative can enter the possible areas that the patient may be found in and the zone of his last track.

- The second target users are the volunteers (helpers), people who wish to help and participate in such caring vocation. When they download the application, they will receive broadcasts about the lost people in their city. The application will use in-app notification system to send broadcasts in a particular city for instance Riyadh.

Before designing the application, a survey was conducted among relatives of Alzheimer's patients to get more insights on their needs and make sure the functions that will be implemented are really helpful for them. Indeed, a survey on a sample of 70 persons concerned by the Alzheimer is 
designed. The survey was of 9 questions and it permits to collect the following information:

- $65 \%$ of the relatives have lost Alzheimer's patient and $38 \%$ from them spends a day or more than a day on searching for the patient which shows the importance of reducing the search time to minimize the risk.

- $20 \%$ from relatives usually find the patient at far range from the neighborhood which indicates the importance of safety area feature that allow relatives to specify area if patient wanders beyond that area an alert text is automatically sent.

- $85 \%$ of relatives do get help from their neighbors, relatives and friends rather than the police. It is noticeable that getting help from the police takes longer time.

- $92 \%$ from the relatives prefer getting a direct communication with the person who found the patient, which indicates the importance of providing this feature in the application.

- $80 \%$ like the idea of providing a mobile application that broadcast Alzheimer's patient information, which indicates the importance of providing this feature.

- $97 \%$ from the relatives did not ever deal with any tracking devices, but $87 \%$ from them think it is user friendly and have accuracy. As long as the majority likes this feature, it will be considered in the application.

- One of relatives suggest providing tracking device placed in the patient's body in places where he/she can't see it in order to not be removed. Another one suggests providing bracelet and connected to the mobile application. In another hand, some relatives talked about the difficulties they are facing in general with Alzheimer's patients. Such discussions and survey's findings helped in providing adequate functionalities in the application.

\section{IMPLEMENTATION}

"Locate me" application is composed of three layers: XML layer, Java layer and PHP layer, as shown in Fig. 1. The XML layer consists of XML files that represent the user interfaces, which means that this layer links the user with the application.

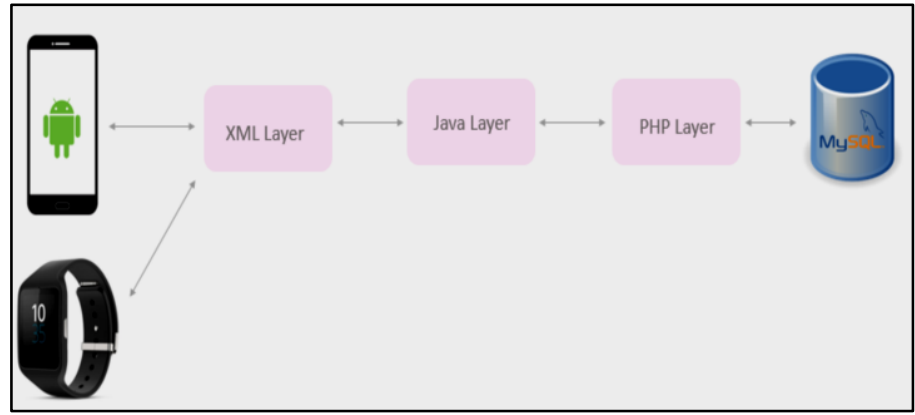

Fig. 1. Locate me layers.
The Java layer consists of Java classes that process user requests that come from XML files. It performs java functions and sends data to the appropriate PHP file by using JSON array, and then views the result in the user interface (XML layer). The PHP layer executes the received requests from Java layer by converting them to MYSQL queries, then performs them on the database and backs the result to Java layer by using JSON array.

Two applications are developed: one for the mobile and another for the watch; each application was integrated through four levels. These levels of integration are as follows.

The first level is concerned by designing the interfaces (XML files) of some system functions, as shown in the figures below (Fig. 2 and 3).

The second level is about programming all functions of the system (Java classes) and linked each one of them with its interface, as shown in Fig. 4 and 5 below.

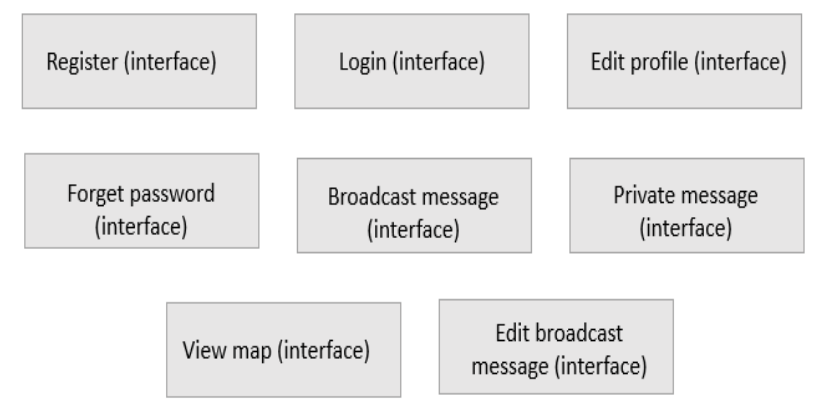

Fig. 2. First level of Locate-me application integration (mobile app).

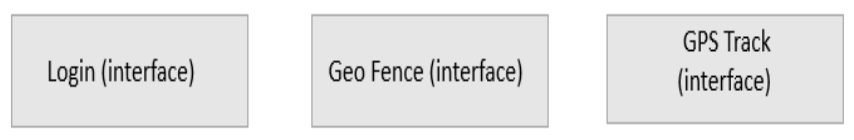

Fig. 3. First level of Locate- me application integration (watch app).

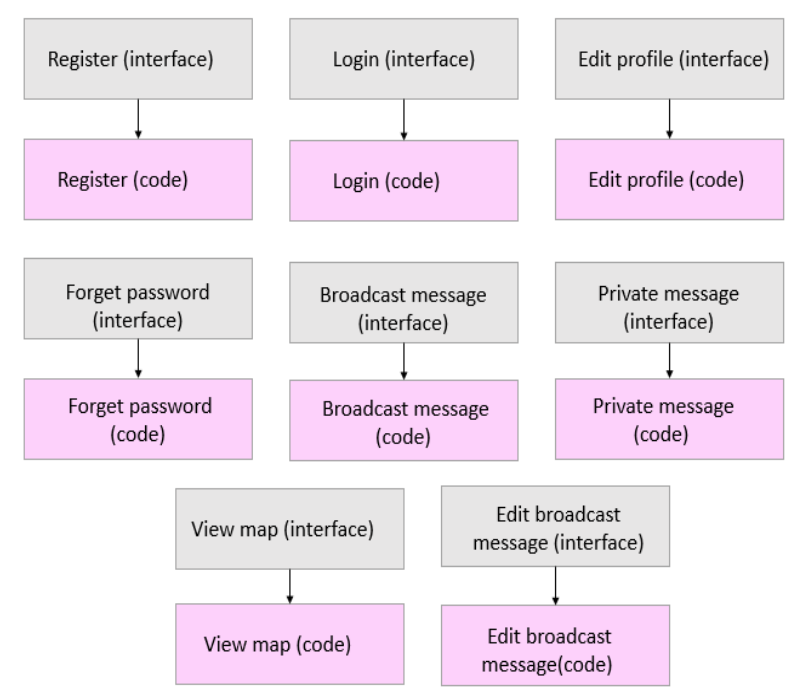

Fig. 4. Second level of locate me application integration (mobile app). 


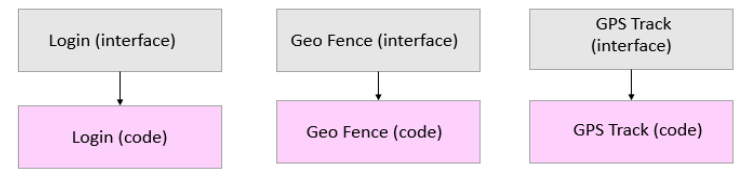

Fig. 5. Second level of locate me application integration (watch app).

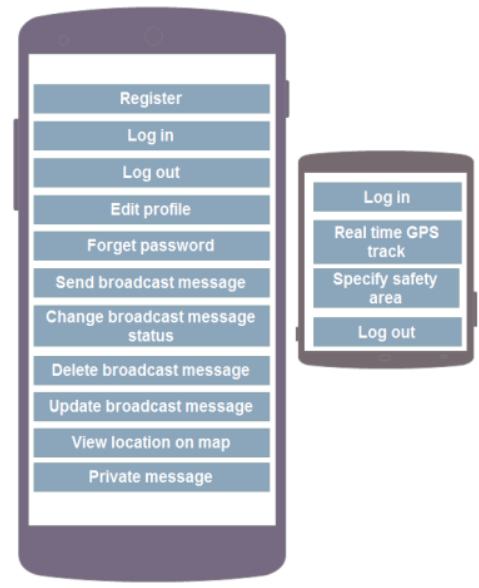

Fig. 6. Third level of Locate-me application integration.

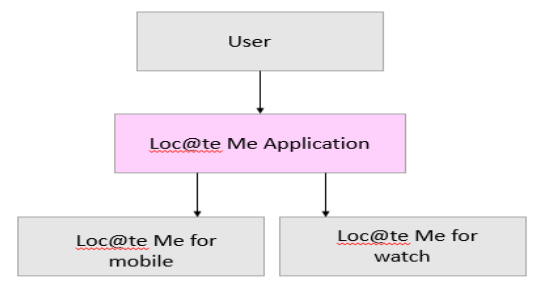

Fig. 7. Fourth level of locate me integration.

In the third level, all functions that belong to each type of users are linked with each other and added the session, as shown in Fig. 6.

In the fourth level, all users' functions are linked with the system. Thus, the system has been fully integrated, as shown in Fig. 7.

\section{APPLICATION FUNCTIONING}

In locate-me, the navigation through different interfaces is shown in Fig. 8.

Fig. 9 illustrates the navigation bar and Fig. 10 shows some interfaces of the application. The role of each function in the navigation bar is described below:

1) This button permits the user to move to the Reports page.

2) This button permits the user to move to the View Location page.

3) This button permits the user to move to the New Broadcast Message page.

4) This button permits the user to move to the Messages page.

5) This button permits the user to move to the Profile page.

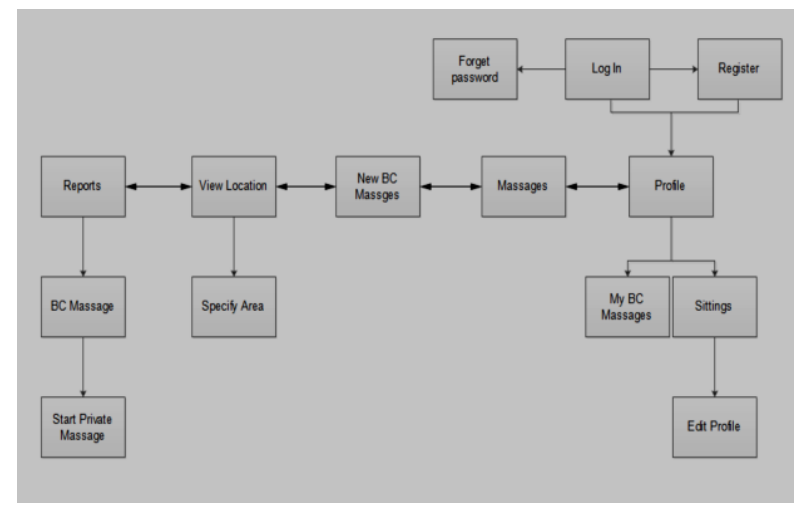

Fig. 8. Locate-me navigation.

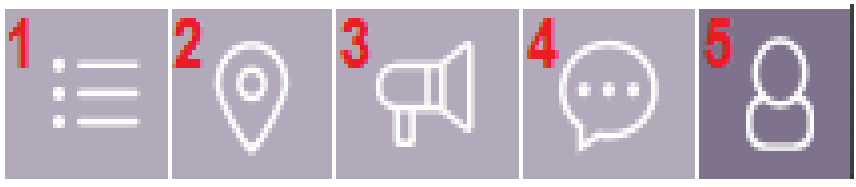

Fig. 9. Navigation bar.

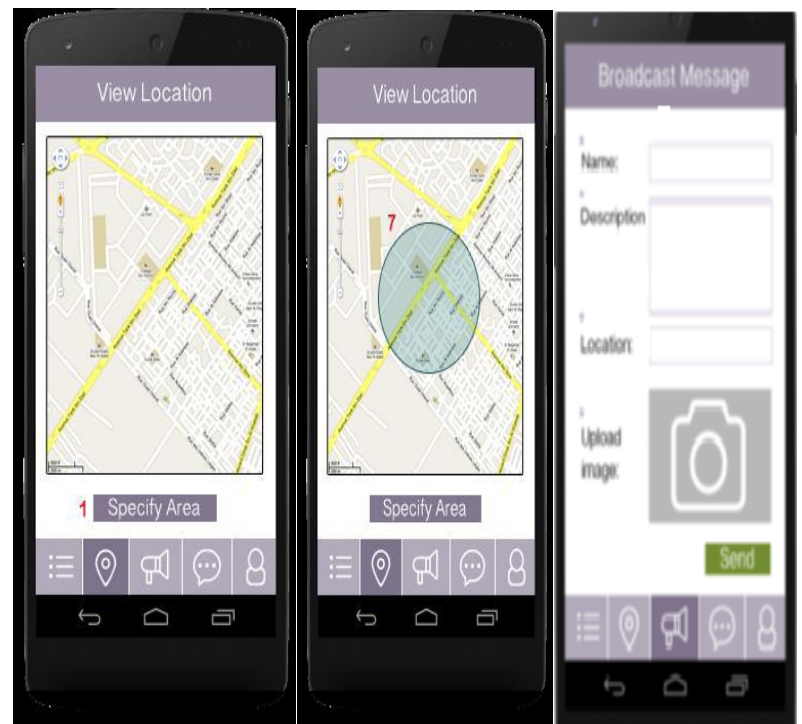

Fig. 10. Some application's interfaces.

In order to track the Alzheimer patient his/her relatives can define a vicinity area to allow them locating and tracking Alzheimer's patient. This tacking is possible by connecting the application with GPS watch that is worn by Alzheimer's patient. When the patient goes beyond the predefined ranges, the relatives are immediately notified by a message on their mobile phone. However, when the connection with the GPS watch is lost and the patient is not further tracked, the application provides an alternative by using instantly in-app notification to broadcast help messages to the application's users to help locating Alzheimer's patient. Relatives can send a photo, a patient description and the latest position coordinates before being out of tracking. According to the previous features the relatives can find Alzheimer's patient in a shorter time. The communication between users and relatives can either be public or private. When discussing with Alzheimer relatives this way of collaboration and assistance is more efficient and more rapid than the traditional ways. 


\section{CONCLUSION}

The suffering life of the Alzheimer's patients and their families and the difficulties that they are facing to go through life is not easy. The main concern about having Alzheimer's patients is keeping them under the sight and supervision without constraining them. In this paper, an android mobile application Locate-me is presented. It has been implemented specially to help Alzheimer's patient's relatives in tracking their patients or finding them in case they were lost through many functionalities.

Locate-me allows Alzheimer's patient's relatives to have a real time tracking for Alzheimer's person who is wearing Sony watch with the ability to define a safety area to notify them if they went beyond the boundary of this area. In addition, the user of the application can interact with other users and ask for help in case of losing the ability to track the patient. They could send a broadcast message in the application containing some important details about the patient, for example his/her last location and the possibility of uploading a photo of the patient.

Other users, who are willing to help, can ask about more details by sending a private message to the relative with the ability to upload an image or sharing their location in case they found the patient. The relatives will receive the messages and they could reply to the senders.
As future work, we are in contact with the Alzheimer association in Riyadh city in order to let them use and test the application. Their feedback will be very beneficial to permit the improvement of "Locate-Me".

\section{ACKNOWLEDGMENT}

This research project was supported by a grant from the "Research Center of the Female Scientific and Medical Colleges", Deanship of Scientific Research, King Saud University.

REFERENCES

[1] https://www.alzheimers.org.uk/site/scripts/documents_info.php?docume $n t I D=100$

[2] https://www.alz.org/national/documents/brochure_basicsofalz_low.pdf

[3] “PocketFinder 2." https://itunes.apple.com/us/app/pocketfinder2/id468281892?mt=8 [Accessed: 21-March-2015].

[4] “Tweri” [Online]. Available: http://www.tweri.com/en-tweri.[Accessed: 21-March-2015].

[5] "Features" http://www.comfortzonecheckin.com/HowItWorksSoftware.aspx

[6] MCJEEL Entertainment Inc. https://itunes.apple.com/us/app/safetysnapp/id843700040?mt=8

[7] Cahill, S., Macijauskiene, J., Nygrd, A., Faulkner, J., Hagen, I.: Technology in dementia care. Technol. Disabil. 19(2), 55-60 (2007)

[8] Raghunath, M.T., Narayanaswami, C.: User interfaces for applications on a wrist watch. Pers. Ubiquit. Comput. 6(1), 17-30 (2002)

[9] "Smartwatch3 SWR50". http://www.sonymobile.com/globalen/products/smartwear/smartwatch-3-swr50 